\title{
Functional mRNA analysis reveals aberrant splicing caused by novel intronic mutation in WDR45 in NBIA patient
}

Josh Willoughby ${ }^{1}$, Celia Duff-Farrier ${ }^{2}$, Archana Desurkar ${ }^{3}$, Manju Kurian ${ }^{4}$, Ashok

Raghavan $^{5}$, Meena Balasubramanian ${ }^{6}$

${ }^{1}$ Sheffield Diagnostic Genetics Service, Sheffield Children's NHS Foundation Trust, UK

${ }^{2}$ Bristol Genetics Laboratory, Pathology Sciences, Southmead Hospital, Bristol, UK

${ }^{3}$ Department of Neurology, Sheffield Children's NHS Foundation Trust, Sheffield, UK

${ }^{4}$ Department of Paediatric Neurology, Great Ormond Street Hospital, London, UK

${ }^{5}$ Department of Radiology, Sheffield Children's NHS Foundation Trust, Sheffield, UK

${ }^{6}$ Sheffield Clinical Genetics Service, Sheffield Children's NHS Foundation Trust, UK

Correspondence to:

Josh Willoughby, Sheffield Diagnostic Genetics Service, Sheffield Children's NHS

Foundation Trust, Western Bank, Sheffield S10 2TH, Phone- +44 114 2717284; Fax- +44

114 2750629, E-mail: josh.willoughby@sch.nhs.uk AND

Dr Meena Balasubramanian, Sheffield Clinical Genetics Service, Sheffield Children's NHS

Foundation Trust, Western Bank, Sheffield, UK, Ph: +44 114 2717025, Fax: +44 114

2737467, E-mail: meena.balasubramanian@nhs.net

Keywords: iron accumulation, WDR45, mRNA analysis, intronic, splicing, mutation, cultured fibroblasts, MRI-brain, in silico analysis

Word count $=2328$ (excl. title page, abstract, references, figures and tables) 


\begin{abstract}
WDR45 gene-associated Neurodegeneration with Brain Iron Accumulation (NBIA), referred to as beta-propeller protein-associated neurodegeneration (BPAN), is a rare disorder that presents with a very non-specific clinical phenotype in children constituting global developmental delay. This case report illustrates the power of a combination of trio exome sequencing, in silico splicing analysis and mRNA analysis to provide sufficient evidence for pathogenicity of a relatively intronic variant in WDR45, and in so doing, find a genetic diagnosis for a 6-year old patient with developmental delay and seizures, a diagnosis which may otherwise have only been found once the characteristic MRI patterns of the disease became more obvious in young adulthood.
\end{abstract}

\title{
INTRODUCTION
}

Mutations in the WDR45 gene are associated with an X-linked dominant form of Neurodegeneration with Brain Iron Accumulation (NBIA) [Haack et al, 2012], referred to as beta-propeller protein-associated neurodegeneration (BPAN). The term NBIA, encompasses several single-gene disorders characterized by abnormal iron deposition in the basal ganglia [Gregory et al 2017].

To date, there are 10 genes established to be associated with NBIA, the three most common forms being caused by mutations in the PANK2 (OMIM 606157), C19orf12 (OMIM 614297), and PLA2G6 (OMIM 603604) genes, which encode mitochondria-associated proteins with no clear link to iron homeostasis. The X-linked WDR45 gene encodes a beta-propeller scaffold protein with a putative role in autophagy [Carvill et al 2017]. Eight of the ten genetically defined types of NBIA are inherited in an autosomal recessive manner. Based on observed 
cases in the population, the prevalence of NBIA is estimated at 1-3/1,000,000 [Gregory et al 2005] of which BPAN constitutes only one to two percent [Horvath 2013].

Typical clinical features of BPAN include early-onset global developmental delay remaining essentially static until young adulthood when there is further neurological deterioration (Parkinsonism, dystonia, and dementia). Seizures and sleep disorders are also a relatively common feature. During the early years, the clinical picture is very non-specific making diagnosis difficult [Gregory et al 2017].

A characteristic pattern observed on MRI in BPAN patients, thought not to become apparent until the further neurological deterioration of young adulthood has occurred, is T1 hyperintensity surrounding a central linear region of signal hypointensity within the substantia nigra and cerebral peduncles. Cerebral and cerebellar atrophy are also observed.

The majority of mutations reported in WDR45 to-date are truncating mutations predicted to result in a loss of function. Mutations at the canonical splice site positions of several WDR45 exons have also previously been reported. Based on the positions of the mutations reported to date, there is no apparent mutational hotspot.

Here we describe how a genetic diagnosis of BPAN was reached in a 6-year old child with a typical non-specific phenotype, via the use of trio exome sequencing, in silico splicing analysis and functional mRNA characterisation demonstrating aberrant splicing as the result of a novel, relatively intronic variant in WDR45.

\section{CLINICAL REPORT}

The proband is the second child of healthy non-consanguineous, White European parents with no significant family history of note. The pregnancy was uncomplicated with normal 
antenatal scans. She was born at term with a birth weight of $4130 \mathrm{~g}$ and was in a good condition immediately after birth. There were no concerns immediately after birth but she was noted to have developmental delay in the first year of life.

At one year of age, she was only sitting unsupported, was not able to stand without support and just about being able to transfer objects. She also developed seizures from 4-months of age, initially managed conservatively but requiring medical management soon thereafter.

This patient was initially referred to the Paediatric Neurology clinic at 10-months of age with developmental delay and seizures. She was evaluated and found to have no focal neurological abnormality and no evidence of regression. An MRI-brain scan at 1-year of age demonstrated abnormal high signal in white matter around dentate nuclei bilaterally with overall decreased white matter bulk with ex-vacuo ventricular dilatation. Investigations at the time included extensive Neurometabolic screen including testing for leukodystrophy and neurodegenerative conditions (VLCFA profile, urine organic and plasma amino acids, purine and pyrimidine assays and isoelectric focussing of transferrins) which were negative. She went on to develop epileptic spasms around 18-months of age and EEG at the time showed documented evidence of hypsarrhythmia. She was diagnosed with epilepsy at 2-years of age and initially trialled with Vigabatrin and steroids; and a trial of pyridoxine all with only a partial response. She was subsequently treated with Topiramate with good response. However, she went on to have intermittent clusters of epileptic spasms and commenced on ketogenic diet therapy with better response. She also developed astigmatism and myopia needing glasses. In terms of her general health, she developed recurrent urinary tract infections needing prophylactic antibiotics and pyelonephritis needing stenting at 3-years of age.

This patient was initially seen in the Genetics clinic at a year of age, when she was noted to have metopic sutural craniosynostosis, deep-set eyes, a small mouth with thin upper lip and 
broad forehead with bilateral low-set ears (Figure 1a-b). Her growth parameters included head circumference $47.5 \mathrm{cms}\left(75^{\text {th }}-91^{\text {st }}\right.$ centile $)$, weight and height $98^{\text {th }}-99^{\text {th }}$ centile. Genetic investigations at the time included arrayCGH, mitochondrial testing, MECP2 and targeted epilepsy and craniosynostosis panel, which were all negative. She was subsequently reviewed again at 3-years of age and noted to have similar findings as before (Figure 1c-d) with some improvement in her development. She was recruited to the Deciphering Developmental Disorders (DDD) Project for trio exome analyses which identified a WDR45 intronic variant.

Further to this result the patient had MRI-brain scan performed at 4-years of age which showed mildly reduced T2 signal in bilateral globus pallidus on SWI and persistent white matter signal changes (Figure 2a-b). Repeat MRI at 5-years of age (Figure 2c-d) demonstrated mineralisation within the dentate nuclei, globus pallidus and substantia nigra, possibly in keeping with NBIA.

Her most recent clinical examination at 6-years of age demonstrated a very sociable young girl with good eye contact, developmental delay with moderate intellectual disability (needing special educational support). She was able to stand with support but had difficulty with her balance; she was crawling but unable to walk with support. She was using a wheelchair, remained non-verbal but had a very happy, friendly personality. She was wearing glasses for astigmatism and myopia but her hearing was reported to be normal. She continued to have multi-focal frequent myoclonic seizures needing treatment with multiple antiepileptic medications including Lamotrigine, Topiramate and more recently started on trial of sodium valproate in addition to the ketogenic diet to attain seizure-control. Her growth parameters included head circumference $52 \mathrm{cms}\left(50^{\text {th }}-75^{\text {th }}\right.$ centile $)$, weight $24.2 \mathrm{~kg}\left(98^{\text {th }}\right.$ centile) and height $111.5 \mathrm{cms}\left(50^{\text {th }}-75^{\text {th }}\right.$ centile $)$. 


\section{MATERIALS AND METHODS}

This patient was recruited to the Deciphering Developmental Disorders (DDD) study, triobased exome sequencing was performed on the affected individual and their parents, as previously described [Wright et al., 2014]. Each affected individual has also had a highresolution analysis for copy number abnormalities using array-based comparative genomic hybridization (aCGH). Putative de novo mutations were identified from exome data using DeNovoGear software [Ramu et al., 2013] and were validated using targeted Sanger sequencing.

In silico splicing analysis: the following in silico tools were utilised, accessed via Alamut Visual v2.9. SpliceSiteFinder-like (http://www.umd.be/HSF/), MaxEntScan (http://genes.mit.edu/burgelab/maxent/Xmaxentscan_scoreseq.html), GeneSplicer (http://www.cs.jhu.edu/ genomics/GeneSplicer/), NNSPLICE (https://omictools.com/nnsplice-tool), Human Splicing Finder (http://www.umd.be/HSF3/). mRNA analysis: Epidermal fibroblasts were received as a confluent monolayer growth in a T25 flask. Cells were washed once using PBS then detached using a Trypsin-EDTA solution and centrifugation at 1500 RPM for 5 minutes. Cells were washed again once in PBS, and RNA extracted from half of the total volume using the RNeasy Mini Kit (Qiagen, Hilden, Germany) on the Qiacube ${ }^{\circledR}$ (Qiagen) as according to the manufacturer's instructions. cDNA was synthesised from $25 \mu \mathrm{l}$ of total RNA using the High Capacity cDNA RT kit (Thermo Fisher Scientific Waltham, MA) and random oligo priming in a total volume of $50 \mu \mathrm{L}(10 \times$ Random Primers 5.0 $\mu \mathrm{L}, 10 \times$ Buffer 5.0 $\mu \mathrm{L}$, dNTPs $(100 \mathrm{~mm}) 2.0 \mu \mathrm{L}$, Multiscribe Reverse Transcriptase $2.5 \mu \mathrm{L}$, RNase Inhibitor $2.5 \mu \mathrm{L}$, RNA $25 \mu \mathrm{L}$, RNase Free Water 8.0 $\mu \mathrm{l}$ ). All PCR was conducted using Phusion® DNA polymerase (New England Biolabs, Ipswich, MA) as according to the manufacturer's instructions, with an annealing temperature of $61^{\circ} \mathrm{C}$ and 
15s extension time. PCR primers were designed using Primer-BLAST. Primers were designed to amplify a region spanning exon 3 to exon 8: Primer sequences for PCR and for sequencing were as follows:

WRD45 fwd: 5' AACAGCCACTTCGAGGAGTG 3'

WDR45 rev: 5' GGAACACTAGCAGTTGCTTC 3'

Products were separated by $3 \%$ agarose gel electrophoresis (50\% Nusieve® (Lonza, Basel, Switzerland): 50\% Agarose MP (Roche, Basel, Switzerland)) using 1× TBE buffer and sized using a 50 bp GeneRuler ladder (Thermo Fisher Scientific).

Amplicons were extracted from agarose gels using a Monarch® DNA Gel Extraction Kit (New England Biolabs) as according to the manufacturer's instructions. Products were sequenced using a BigDye® Terminator kit v3.1 (Applied Biosystems) and analysed using an ABI 3730 DNA sequencer (Applied Biosystems). ABI sequence scanner v1.0 was used for electropherogram analysis.

\section{RESULTS}

DNA analysis: Trio exome sequencing through the DDD Project identified the de novo heterozygous ChrX(GRCh37):g.48934430T>C, c.236-18A>G variant in intron 5 of the WDR45 gene (variant nomenclature according to Human Genome Variation Society guidelines with reference transcript NM_007075.3). This particular variant has not been reported previously in the literature.

In silico splicing analysis: in silico tools (see methods section) indicated this variant may interfere with normal splicing at the exon 6 splice acceptor site. Although the NNSPLICE tool failed to offer a prediction, the other 4 tools all indicated activation of a cryptic exon 6 
acceptor splice site at c.236-17 with abolition of the natural acceptor splice site at c.236. This variant was confirmed by Sanger sequencing on a genomic DNA sample from the proband, and Sanger sequencing on parental samples confirmed the de novo status of this variant. This variant was completely absent from population databases (gnomAD, 1000 genomes project). Given the variant's de novo status, the in silico prediction, the absence from population databases and the phenotype and MRI-imaging being consistent with the genotype, we undertook mRNA functional analysis on a blood sample and cultured fibroblasts in search of further evidence of an effect on splicing.

mRNA Analysis: After PCR across exons 3 to 8, gel electrophoresis revealed two major bands from both patient and wild-type control samples (See Figure 3.). All samples showed a band corresponding to an amplicon of approx. 500bp in size, consistent with that expected from the wild-type (WT) transcript. Sanger sequencing of this band from the K562 control (Figure 3; lane 6) was consistent with the WDR45 reference transcript NM_001029896.1, which lacks 3 nucleotides at the beginning of exon 6 relative to the NM_007075.3 transcript. The corresponding band from the affected patient (Figure 3; lane 1) appeared fainter and less discreet than that of the WT controls and sequencing did not produce a reliably interpretable result, other than to determine that it was WDR45-derived.

A secondary band corresponding to an amplicon of approx. 400bp in size could be seen in the PCR product generated from the affected patient (Figure 3; lane 1). Sanger sequencing of this band revealed an aberrant transcript exhibiting exon 5 (75bp) skipping with the incorporation of 17bp of intron 5 (Figure 4). A secondary band corresponding to an amplicon of approx. 375 bp could also be seen in the PCR product generated from the WT controls (Figure 3; lanes 2-6). Sanger sequencing of the secondary band from the K562 control showed it to comprise a transcript exhibiting exon 5 skipping and lacking the 3 nucleotides at the beginning of exon 6 as does the NM_001029896.1 reference transcript, but not containing the 
$17 \mathrm{bp}$ of intron 5 seen in the aberrant transcript from the patient sample. This indicates that the skipping of exon 5 likely represents a normal variant transcript not related to the effect of the c.236-18A >G mutation. Sanger sequencing of total PCR product from the patient sample was also carried out and results showed the sequence corresponding to the aberrant transcript to be by far the predominant PCR product present.

The presence of this aberrant transcript may indicate that it does not undergo nonsensemediated decay, resulting in the production of a truncated protein product, however further analysis to demonstrate the presence of a truncated protein would be required to establish this. Other investigators have demonstrated, from cells of patients with WDR45 truncating mutations, no evidence of truncated protein product, suggesting the mutant proteins are structurally unstable and undergoes degradation [Saitsu et al 2013].

\section{DISCUSSION}

For changes at canonical splice site positions ( $\pm 2 \mathrm{bp}$ from the ends of exons), it is generally safe to assume a deleterious effect unless there is evidence to the contrary, but variants that lie deeper within an intron are much less likely to have a significant impact on splicing and so require further evidence to support a conclusion of pathogenicity, and for a clinical diagnosis in silico predictions cannot be relied upon alone.

The c.236-18A $>\mathrm{G}$ variant, although relatively intronic, was close enough to the exon-intron boundary to be picked up via the particular exome sequencing protocol used by the DDD Project. However, the extent of intronic sequence coverage achieved for any given gene by exome sequencing is variable and dependent on the protocol used, amongst other factors. 
Cases such as this serve as a reminder of the potential importance of this coverage, particularly the context of recent research [Vaz-Drago et al 2017] and illustrate the utility of in silico splicing analysis to help identify variants with a potential to disrupt splicing.

Though end-point PCR was used for the cDNA analysis, which does not provide an accurate indication of relative transcript abundance, our results do suggest the possibility of skewing of X-inactivation in favour of the mutant allele. Other investigators have shown similar results suggestive of skewed X-inactivation [Saitsu et al 2013] suggesting this may form an inherent aspect of the disease mechanism. The number of female patients reported to date significantly outweighs that of males but the phenotype is similar between the sexes. Haack et al. (2012) have previously concluded that males with WDR45 mutations must be somatic mosaic, which was demonstrated in 1 affected male. It is thought therefore that males with germline WDR45 mutations may be nonviable. However, there are now several reports of surviving males with germline WDR45 variants [Takano et al 2017; Redon et al 2017].

This case also serves as another example of the power of trio exome sequencing to find a relatively early diagnosis for a patient with very non-specific clinical features, however it also demonstrates that for some variants further work is required in order to provide sufficient evidence for pathogenicity beyond de novo status.

\section{ACKNOWLEDGEMENTS}

We would like to thank the family for agreeing to include their details. 
Author Roles: All authors contributed to preparation and critical review of manuscript; MB:

Study design; recruitment of patient; phenotyping; JW and CD-F: writing up paper and mRNA analysis studies; AR: radiology input; AD: clinical data.

\section{COMPETING INTERESTS}

No competing interest to declare.

\section{REFERENCES}

Carvill GL, Liu A, Mandelstam S, Schneider A, Lacroix A, Zemel M, McMahon JM, BelloEspinosa L, Mackay M, Wallace G, Waak M, Zhang J, Yang X, Malone S, Zhang YH, Mefford HC, Scheffer IE. (2017). Severe infantile onset developmental and epileptic encephalopathy caused by mutations in autophagy gene WDR45. Epilepsia. 2017 Nov 24. doi: 10.1111/epi.13957.

Gregory, A., \& Hayflick, S. J. (2005). Neurodegeneration with brain iron accumulation. Folia Neuropathologica / Association of Polish Neuropathologists and Medical Research Centre, Polish Academy of Sciences, 43(4), 286-296.

Gregory A, Kurian MA, Haack T, Hayflick SJ, Hogarth P. Beta-Propeller Protein-Associated Neurodegeneration. In: Adam MP, Ardinger HH, Pagon RA, Wallace SE, Bean LJH, Stephens K, Amemiya A, editors. GeneReviews ${ }^{\circledR}$ [Internet]. Seattle (WA): University of Washington, Seattle; 1993-2018. 2017 Feb 16

Haack, T. B., Hogarth, P., Kruer, M. C., Gregory, A., Wieland, T., Schwarzmayr, T., ... Hayflick, S. J. (2012). Exome Sequencing Reveals De Novo WDR45 Mutations Causing a 
Phenotypically Distinct, X-Linked Dominant Form of NBIA. American Journal of Human Genetics, 91(6), 1144-1149.

Horvath R. (2013). Brain iron takes off: a new propeller protein links neurodegeneration with autophagy. Brain 136(6):1687-91.

Ramu, A., Noordam, M. J., Schwartz, R. S., Wuster, A., Hurles, M. E., Cartwright, R. A., \& Conrad, D. F. (2013). DeNovoGear: de novo indel and point mutation discovery and phasing. Nature Methods, 10(10), 985-987.

Redon S, Benech C, Schutz S, Despres A, Gueguen P, Le Berre P, Le Marechal C, Peudenier S, Meriot P, Parent P, Ferec C. (2017). Intragenic deletion of the WDR45 gene in a male with encephalopathy, severe psychomotor disability, and epilepsy. Am J Med Genet A, 173(5):1444-1446.

Saitsu, H., Nishimura, T., Muramatsu, K., Kodera, H., Kumada, S., Sugai, K., KasaiYoshida, E., Sawaura, N., Nishida, H., Hoshino, A., Ryujin, F., Yoshioka, S., and 9 others. De novo mutations in the autophagy gene WDR45 cause static encephalopathy of childhood with neurodegeneration in adulthood. Nature Genet. 45: 445-449, 2013

Takano K, Goto K, Motobayashi M, Wakui K, Kawamura R, Yamaguchi T, Fukushima Y, Kosho T. (2017). Early manifestations of epileptic encephalopathy, brain atrophy, and elevation of serum neuron specific enolase in a boy with beta-propeller protein-associated neurodegeneration. Eur J Med Genet, 60(10):521-526.

Vaz-Drago R., Custódio N., Carmo-Fonseca M. (2017). Deep intronic mutations and human disease. Human Genetics, [Epub ahead of print]

Wright, C. F., Fitzgerald, T. W., Jones, W. D., Clayton, S., McRae, J. F., van Kogelenberg, M., ... on behalf of the DDD study. (2015). Genetic diagnosis of developmental disorders in 
the DDD study: a scalable analysis of genome-wide research data. Lancet, 385(9975), 13051314.

\section{FIGURE LEGENDS}

Figure 1a-b: Photographs of patient (frontal and profile) at 1 year of age demonstrating metopic synostosis, flat nasal bridge with a pointed chin, broad forehead and bilateral low-set ears.

Figure 1c-d: Photographs of patient at age 3 demonstrating evolution of facial dysmorphism of age.

\section{Figure 2a-d:}

2a and b: Selected 3T MRI-brain images done at 4-years of age demonstrating low signal intensity on susceptibility weighted imaging. Image involving substantia nigra and lentiform nucleus.

2c and d: 3T MRI done at 5-years of age showing progressive increase in the low signal intensity on susceptibility weighted imaging involving substantia nigra and lentiform nucleus secondary to iron deposition.

Figure 3: 3\% agarose gel displaying WDR45 cDNA exon 3-8 PCR products. Lane 1; affected c.[236-18A>G] patient epidermal fibroblast derived cDNA, Lane 2; Wildtype (WT) patient control 1 cDNA derived from epidermal fibroblasts, Lane 3; WT patient control 2 cDNA derived from EDTA peripheral blood, Lane 4; WT patient control $1 \mathrm{cDNA}$ derived from PAX gene peripheral blood, Lane 5; WT patient control 2 cDNA derived from PAX 
gene peripheral blood, Lane 6; K562 cell line derived cDNA control, Lane 7; negative control $\mathrm{H}_{2} \mathrm{O}$.

Figure 4: Schematic to illustrate the effect of the WDR45 c.[236-18A>G] variant on mRNA transcription. A; genomic sequence (exons represented by coloured boxes, successive introns represented by lines), B; aberrantly spliced mRNA transcript displaying exon 5 skipping and incorporation of $17 \mathrm{bp}$ of intron 5, C; Electropherogram trace obtained from the Sanger sequencing of mRNA transcript B. 